\title{
Review of current Lasers in the Management of Glaucoma
}

\author{
Carey Pate, Sunil Deokule
}

Department of Ophthalmology and Visual Sciences, University of Kentucky, 740 S Limestone, Lexington, KY, USA

\begin{abstract}
Lasers are used extensively in the treatment of glaucoma. This review is targeted to develop a basic understanding of how a laser functions and affects the targeted ocular tissue. The clinical decision making and optimal parameters of the same are discussed here. The review also aims to highlight the latest developments in this field which is fast evolving.
\end{abstract}

\section{INTRODUCTION}

Lasers are used extensively within many specialties of ophthalmology and have become an important piece of our armamentarium in the treatment of glaucoma. It is critical that ophthalmologists, who deliver this crucial therapy, develop a basic understanding of how a laser functions and affects the targeted ocular tissue. This will help us to improve our clinical decision making and allow us to synthesize new laser technology that becomes available.

\section{LASER MECHANISM AND SPECTRUM}

Laser light is monochromatic, coherent, and directional, allowing a high amount of energy to be delivered to precise area. The primary substance or "lasing medium" is contained within a reflective cylindrical chamber. When energy is pumped into the chamber with either DC current (gas lasers) or a flash-lamp (solid state lasers), the medium is stimulated to emit photons of specific wavelengths. Those photons that are released parallel to the length of the cylinder begin to reflect off the mirrors at either end of the tube, amplifying and stimulating the lasing medium at each pass. This leads to an avalanche of photons that have the same wavelength (monochromatic), are in-step (coherent), and are oriented in the same direction (wellcollimated). Since one of the mirrors is only partially reflective, a beam of photons is released at the end of the tube. The substance of the lasing medium determines the specific type of laser and can be a gas such as argon or krypton or a solid such as an Nd:YAG crystal. Whereas gas occupies the space inside the chamber, solid state lasers are comprised of ions (i.e. neodymium) doped into a crystal (yttrium-aluminum-garnet) that allows the photons to reflect freely back-and-forth through the chamber.
Any laser that is used to penetrate the external surface of the eye and act on intraocular tissue must emit light waves in the visible spectrum or near infrared (400 nm to $1200 \mathrm{~nm}$ ). Ultraviolet light is mostly absorbed by the cornea and sclera and infrared wavelengths above 1200 nanometers are absorbed well by water, making them unable to penetrate the ocular surface. This is the reason that excimer (193 nm ultraviolet) and carbon dioxide (10600 nm infrared) are effective on surface tissue for sublimation and cutting, respectively. Within the visible light and near infrared spectrum, two basic types of lasers have been employed to target intraocular tissue: thermal lasers and disruptive lasers (Fig. 1).

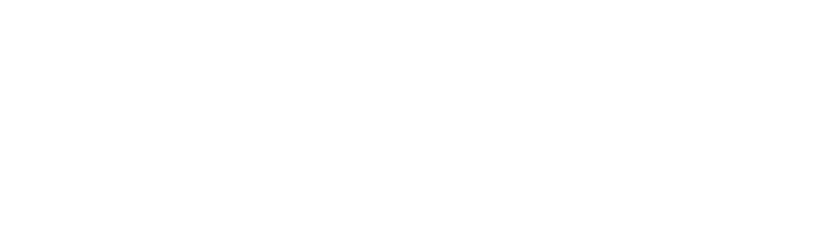

Fig. 1: Glaucoma laser image

\section{THERMAL VERSUS DISRUPTION LASERS}

Thermal lasers cause photocoagulation by increasing the temperature of the tissue and causing the proteins to denature. In the eye, the most common target is black melanin pigment which absorbs light across the entire visible spectrum (although shorter wavelengths are more completely absorbed). If blood vessels need to be targeted, yellow light around 570 nanometers is ideal due to the absorption spectrum of hemoglobin. Using green light will primarily target the uveal pigment with less effect on blood vessels. While continuous wave lasers emit a circular shaped beam, a small surrounding ring of "thermal blooming" occurs as heat dissipates into the nearby tissue. Thermal lasers operate in "continuous wave" mode, as opposed to the single burst of a Q-switched photodisruption laser. For this reason, a specific interval of exposure (usually set in milliseconds) must be programmed before firing the laser and the power must be set in milliwatts rather than Joules. The most common types of continuous wave thermal lasers used in ophthalmology are the 
argon, krypton, diode, and Nd:YAG. Unlike its Q-switched counterpart, this is a continuous wave Nd:YAG laser that is frequency doubled and emits a green $532 \mathrm{~nm}$ beam. Therefore, it functions similar to the krypton or argon lasers and causes tissue photocoagulation.

Disruption lasers operate in the visible light spectrum or near infrared are able to penetrate the clear cornea and target specific intraocular structures. A laser that operates in "Qswitched” mode produces a very short, high energy laser beam burst as higher energy photons are suddenly released from the lasing chamber. When fired, a small area of high temperature and pressure at the laser focus contracts and releases a destructive shockwave, causing photodisruption via optical breakdown of surrounding tissue. In contrast to the thermal laser, no absorption of light is necessary and the disruption phenomenon occurs without thermal tissue damage. The major disruption laser used in ophthalmology is the Q-switched $\mathrm{Nd}$ :YAG laser that operates at $1064 \mathrm{~nm}$ or is frequency doubled to operate at $532 \mathrm{~nm}$ (SLT). Because the interval of laser exposure cannot be changed, only the total energy burst in Millijoules needs to be toggled before use.

In clinical use, thermal lasers target and photocoagulate pigmented tissue. In the eye, uveal tissue, retinal pigment epithelium, and pigmented trabecular meshwork absorb visible spectrum laser light. In contrast, disruptive lasers cause a shockwave blast at the laser focal point leading to breakdown of nearby tissue, whether it is pigmented or not. Whereas thermal lasers must be absorbed by the target tissue, photodisruptive lasers create an independent optical shockwave that damages nearby tissue. Understanding this principle is elementary in understanding the function of different laser types.

Laser therapy plays a critical role in the management of glaucoma. Currently several lasers are available that target different ocular structures.

\section{TRABECULAR MESHWORK}

Pigment in the trabecular meshwork makes the visible spectrum thermal laser ideal to target the molecules of melanin. In thermal laser trabeculoplasty (TLT), the pigmented trabecular meshwork can be targeted with any number of lasers: argon laser (ALT), diode laser (DLT), krypton laser (KLT), or continuous wave $532 \mathrm{~nm}$ Nd:YAG laser. Thermal laser photocoagulation of the trabecular meshwork has been performed since the 1970's with argon laser trabeculoplasty (ALT) first being described by Wise et al in 1979. ${ }^{1}$ Over the years ALT became the "gold standard" in laser trabeculoplasty. Scanning electron microscopy studies reveal that an argon laser creates a crater in the trabecular meshwork with surrounding evidence of coagulative damage. ${ }^{2}$ Presumably, the photocoagulative tissue response and enlarged trabecular channels results in increased aqueous outflow and lowering of intraocular pressure. ${ }^{3}$ This procedure is usually performed once or twice in each patient due to the concern over permanent alteration of the trabecular meshwork.

Photodisruption of the pigmented trabecular meshwork can also be achieved through the use of a Q-switched, frequency doubled Nd:YAG laser that operates at $532 \mathrm{~nm}$ and targets melanin. Selective laser trabeculoplasty (SLT) specifically targets the pigment in the trabecular meshwork at much lower energy levels than those used for thermal laser trabeculoplasty (i.e. ALT). ${ }^{4}$ In theory this makes precise aiming of trabecular meshwork unnecessary. Kramer et al and Cvenkal et al described noncoagulative changes in the trabecular beams after SLT that, while present, were much less marked than after ALT. ${ }^{5}$ SLT delivers a short pulse duration of 2-3 nanoseconds, minimizing the heat conversion and collateral tissue damage. For this reason, SLT laser offers a gentle alternative to traditional thermal laser trabeculoplasty and may be repeatable.

Recently, some newer lasers are being studied that merit mention. A titanium sapphire laser trabeculoplasty has been described which utilizes pulses that are 1000 times shorter (8 micro seconds) than conventional thermal lasers while remaining 1000 times longer than the nanosecond Q-switched Nd:YAG (SLT). This laser has recently been FDA approved for a multicenter clinical trial and could provide a hybrid thermal/ disruption laser. ${ }^{6}$ Similarly, micropulse laser trabeculoplasty (MLT) $810 \mathrm{~nm}$ near-infrared diode laser is theorized to target melanin in the trabecular meshwork without causing coagulative damage. It does this by delivering the laser energy in a series of short pulses over a millisecond, keeping the thermal damage to a minimum. Early investigations are underway to determine if these will provide an alternative to ALT or SLT. ${ }^{7,8}$

Similarly, excimer laser trabeculotomy (ELT) can be performed independently or in conjunction with cataract surgery. ${ }^{9}$ Since ultraviolet light is blocked by the cornea and sclera, an endoscope guided excimer ultraviolet laser is used to sublimate portions of the trabecular meshwork. The nature of this cool excimer lasers allow very precise destruction of the trabecular meshwork without thermal damage, similar to the excimer lasers used in LASIK corneal ablation. We await the clinical results of ELT.

\section{IRIS}

In laser peripheral iridotomy (LPI), a thermal laser can be used to target iris pigment. In clinical practice, argon laser is most commonly used as pretreatment before using a photodisruptive Nd:YAG laser in patients with a heavily pigmented iris. Presumably, the heavier the pigmentation of the iris, the more effective a thermal laser would be in creating the iridotomy. Hemorrhage from iris vessels occurs less often in this technique than with the photodisruptive YAG laser, likely from the photocoagulative effect of thermal laser on blood vessels. Alternatively, a standard $1064 \mathrm{~nm}$ q-switched Nd:YAG laser is 
very effective in creating a peripheral iridotomy, with or without the aid of pretreatment with a thermal laser. Care must be taken to get the focus of the beam at the iris plane as a $1064 \mathrm{~nm}$ beam will penetrate pigmented iris tissue and could potentially be harmful to zonules if the micro explosions occur posteriorly, out of the practitioner's view.

In a laser iridoplasty, a thermal laser is used to contract the peripheral iris away from the angle, opening trabecular meshwork to allow increased outflow. Long duration, large spot, low power burns are ideally placed in the peripheral one-third of the iris for best effect. ${ }^{10}$ Although the effect of laser iridoplasty appears to be transient, thermal laser iridoplasty remains a valuable tool to draw iris away from the angle.

\section{CILIARY BODY}

Diode laser contact cyclophotocoagulation can be used in cases of refractory glaucoma to attempt to ablate the ciliary body through the sclera. It has been shown to decrease intraocular pressure in many cases and is potentially a safer option than cyclocryotherapy for cases of refractory glaucoma. ${ }^{11}$ Although light in the visible spectrum can penetrate the sclera, it is scattered somewhat, lessening the effect on the pigmented ciliary body. Further transmission through sclera also depends on scleral thickness, pressure on the sclera, and angle of the probe tip. Consequently, retreatments are frequently required.

Thermal diode laser endocyclophotocoagulation (ECP) of the ciliary body was first described by Uram, and is often performed at the time of cataract surgery or in pediatric glaucoma. ${ }^{12}$ A fiber optic camera is combined with a cyclodestructive laser that enables direct visualization of the whitening and shrinkage of the ciliary body. ECP results in decreased aqueous production and intraocular pressure at low energy levels with less collateral damage than transcleral laser techniques. However, it is associated with significant postoperative complications, anterior chamber inflammation in particular. $^{13}$

\section{OTHER USES FOR LASERS}

In clinical practice, many other uses for lasers have been found to aid in treatment of numerous patient conditions. In malignant glaucoma, it is possible to target the anterior hyaloid face with a disruptive Nd:YAG laser in order to alter the posteriorly directed flow of aqueous humor. In a postoperative suturolysis, targeting a black nylon suture with the argon laser is a safe and effective method for lysing the suture and increasing transscleral outflow. Alternately, a disruptive Nd:YAG lasers are equally effective and might be advantageous in lighter color sutures. In neovascular glaucoma, using any number of thermal lasers for pan-retinal photocoagulation is a critical step to decrease VEGF levels and attempt to cause angle neovascularization to regress. In another interesting procedure, a micro filter from the trabecular meshwork into the subconjunctival space can be created with a disruptive Nd:YAG goniopuncture after creating a deep sclerotomy. ${ }^{14}$

\section{THE FUTURE OF LASERS}

As we look to the future, advances in femtosecond laser technology will allow controlled, methodical, nonthermal destruction of ocular tissue, similar to that used to create laser flaps in LASIK surgery. This could eventually be combined with advanced ultrasound techniques to program destruction of the ciliary body or trabecular meshwork without it being directly visualized. The remarkable technology that is at our disposal provides optimism about the future possibilities of laser therapy. Since medical treatment of glaucoma is limited, lasers have become an essential tool in the ophthalmologists' management of this complex disease. We must continue to expand our understanding and use of lasers to offer better care to our patients.

\section{REFERENCES}

1. Wise JB, Witter SL. Argon laser therapy for open angle glaucoma: A pilot study. Arch Ophthalmol 1979;97:319-22.

2. Kramer TR, Noecker RJ. Comparison of the morphologic changes after selective laser trabeculoplasty and argon laser trabeculoplasty in human eye bank eyes. Ophthalmology 2001;108:773-79.

3. Van Bukir K. Pathophysiology of laser trabeculoplasty. Surv Ophthalmol 1989;33:264-72.

4. Barkana Y, Belkin M. Selective laser trabeculoplasty. Surv Ophthalmol 2007;52:634-54.

5. Cvenkel B, Hvala A, Drnovsek-Olup B, Gale N. Acute ultrastructural changes of the trabecular meshwork after selective laser trabeculoplasty and low power argon laser trabeculoplasty. Lasers Surg Med 2003;33:204-08.

6. National Institute of Health: http://clinicaltrials. gov/ct2/show/ NCT00145535. SOLX Titanium Sapphire Laser (TiSaLT): Identifier: NCT00145535.

7. Goldenfeld M, Melamed S, Simon G, et al. Titanium: Sapphire laser trabeculoplasty versus argon laser trabeculoplasty in patients with open-angle glaucoma. Ophthalmic Surg Lasers Imaging 2009;40:264-69.

8. Detry-Morel M, Muschart F, Pourjavan S. Micropulse diode laser ( $810 \mathrm{~nm}$ ) versus argon laser trabeculoplasty in the treatment of open-angle glaucoma: Comparative short-term safety and efficacy profile. Bull Soc Belge Ophtalmol 2008;308:21-28.

9. Wilmsmeyer S, Philippin H, Funk J. Excimer laser trabeculotomy: A new, minimally invasive procedure for patients with glaucoma. Graefes Arch Clin Exp Ophthalmol 2006;244:670-76. Epub 2005 Oct 19.

10. Ritch R, Tham CC, Lam DS. Argon laser peripheral iridoplasty (ALPI): An update. Surv Ophthalmol 2007;52:279-88. 
11. Bloom PA, Tasi JC, Sharma K, et al. “Cyclodiode”. Transscleral diode laser cyclophotocoagulation in the treatment of advanced refractory glaucoma. Ophthalmology 1997;104:150819; discussion 1519-20.

12. Uram M. Ophthalmic laser micro endoscope ciliary process ablation in the management of neovascular glaucoma. Ophthalmology 1992;99:1823-28.

13. Lima FE, Magacho L, Carvalho DM. A prospective, comparative study between endoscopic cyclophotocoagulation and the Ahmed drainage implant in refractory glaucoma. J Glaucoma 2004;13:233-37.

14. Mermoud A, Karlen ME, Schnyder CC, Nd: Yag goniopuncture after deep sclerectomy with collagen implant. Ophthalmic Surg Lasers 1999;30:120-25.

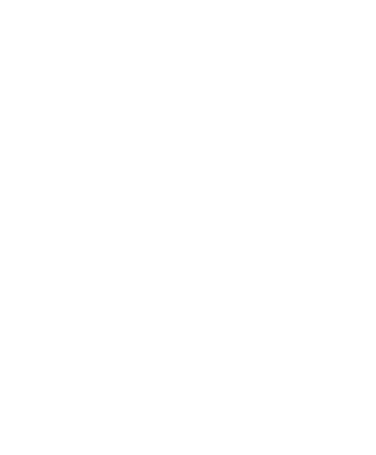

Carey Pate (rena3delta@aol.com)

The more powerful and original a mind, the more it will incline towards the religion of solitude. 